\title{
Polarization switching demonstration using crossed-planar undulators in a seeded free-electron laser
}

\author{
Haixiao Deng, Tong Zhang, Lie Feng, Chao Feng, Bo Liu, Xingtao Wang, Taihe Lan, \\ Guanglei Wang, Wenyan Zhang, Xiaoqing Liu, Jianhui Chen, Meng Zhang, \\ Guoqiang Lin, Miao Zhang, Dong Wang, and Zhentang Zhao* \\ Shanghai Institute of Applied Physics, Chinese Academy of Sciences, \\ Shanghai, 201800, People's Republic of China \\ (Received 18 November 2013; published 18 February 2014)
}

\begin{abstract}
Polarization switching of light sources is required over a wide spectral range to investigate the symmetry of matter. In this paper, we report the first experimental demonstration of the crossed-planar undulators technique at a seeded free-electron laser, which holds great promise for the full control and fast switching of the polarization of short-wavelength radiation. In the experiment, the polarization state of the coherent radiation at the second harmonic of the seed laser is switched successfully. The experimental results confirm the theory and pave the way for applying the crossed-planar undulators technique for seeded $\mathrm{x}$-ray free-electron lasers.
\end{abstract}

PACS numbers: 41.60.Cr

To satisfy the dramatically growing demand for photon sources in biology, chemistry, physics, and the material sciences, high-intensity ultrashort radiation pulses with tunable wavelength are being routinely delivered to users by synchrotron radiation (SR) light sources and freeelectron lasers (FELs) worldwide [1]. With the advent of $\mathrm{x}$-ray FELs in the last 10 years [2-5], a new era of x-ray science has arrived. Currently, the light source community is continuing to develop more sophisticated schemes in pursuit of, e.g., fast polarization switching [5-17], full coherence [18-24], compact x-ray configurations [25-28], and multicolor operation [29-30].

Polarization describes the orientation of the light's electric field at a point in space over one oscillation period. In free space, the electric field of a polarized light may be oriented in a single direction, i.e., linear polarization, or it may rotate as the wave travels, i.e., circular or elliptical polarization. As is well known, SR and FEL light sources usually involve a relativistic electron beam passing through a transverse periodic magnetic field, i.e., the undulator, and generating fully polarized radiation from the infrared to hard x-ray regions. Because of the coupling between the radiation field and the electron motion in the undulator, the polarization state of the radiation is determined by the undulator magnetic field distribution. While a planar undulator only generates linearly polarized light, an elliptically polarized undulator (EPU) is capable of providing radiation pulses with arbitrary polarization.

\footnotetext{
*Corresponding author. zhaozhentang@sinap.ac.cn

Published by the American Physical Society under the terms of the Creative Commons Attribution 3.0 License. Further distribution of this work must maintain attribution to the author $(s)$ and the published article's title, journal citation, and DOI.
}

Light with well-defined polarization is attractive for experiments that aim at exploring the local symmetry of samples, and has become a powerful tool for studying the electronic and magnetic properties of matter. To enhance the signal-to-noise ratio and improve the sensitivity to weakly polarization-dependent signals, one of the recent and growing demands from users is for modulated fast polarization switching at rates from $10 \mathrm{~Hz}$ up to $1 \mathrm{kHz}$ [31-33], e.g., between left-handed and right-handed circular polarization or between horizontal and vertical linear polarization or other combinations. However, the traditional light polarization control is accomplished by the mechanical adjustment of the phase between the EPU magnets, a process which lasts from one to many seconds depending on the magnitude of the desired phase changes in polarization. Therefore, multiundulator techniques have been developed for high-repetition rate polarization switching: (1) Two canted EPUs simultaneously deliver two angularly distinct photon beams with different polarization states, and the fast polarization switching is accomplished by alternately selecting one of the two angularly separated photon beams with a mechanical chopper [15]. (2) The electron beam is actively switched between two EPUs by a fast kick system and is thus capable of delivering one radiation pulse at a time with different polarization [16]. (3) Adjusting the phase between two successive EPUs changes the intensity of circular polarized radiation and thus allows fast polarization switching from circular to linear in a hybrid undulators system [17]. In all of the above-mentioned methods, the circularly polarized radiation is generated from EPUs, and the fast switching can be accomplished between only two preset polarization handedness.

An alternative solution for arbitrarily polarized light is the scheme of crossed-planar undulators, which has been proposed for SR [7] and FEL light sources [8]. It is based 


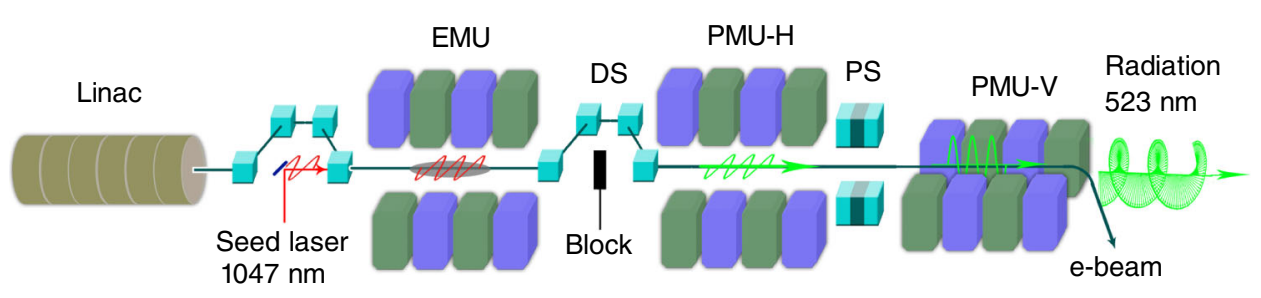

FIG. 1 (color online). Schematic of the polarization switching experiment at SDUV-FEL.

on the interference of horizontal and vertical radiation fields generated from two planar undulators in a crossed configuration, seen in Fig. 1. A pulsed electromagnetic phase shifter between the crossed undulators is used to delay the electron beam and hence to control the final polarization of the radiation. The advantage of the crossedplanar undulators is an arbitrary, programmable, and fast polarization switching. A series of crossed-planar undulators experiments for incoherent SR light source have been carried out at BESSY [14]. The key point of the crossedplanar undulators technique is to make the electromagnetic radiation pulses in the horizontal and vertical axes as close to identical as possible, including the amplitude and the phase. Because of the coherent length of FEL radiation, various approaches using crossed-planar undulators for polarization control have been proposed for modern FELs [8-13]. For self-amplified spontaneous emission (SASE) FELs, the optimum point to implement the crossed undulators is just before power saturation, such that the density modulation induced by the SASE process remains nearly constant while passing through the crossed undulators. However, the intrinsic spiky structure and the shot-to-shot fluctuation of SASE may degrade the FEL polarization performance. While for the seeded FELs, the strong and controllable density modulation introduced by the interaction between the electron beam and the optical seed laser could result in stable and fully coherent output radiation. Thus with the crossed-planar undulators technique, excellent polarization control performance is expected in seeded FELs.

In this paper, we report the first experimental demonstration of polarization switching using the crossed-planar undulators technique, at the Shanghai deep ultraviolet free-electron laser (SDUV-FEL) test facility at Shanghai Institute of Applied Physics [34]. The experiment is a proofof-principle experiment of the crossed-planar undulators technique, which uses the $1047 \mathrm{~nm}$ seed laser in the modulator and the polarization switching of the $523 \mathrm{~nm}$ radiation, i.e., the second harmonic of the seed laser was successfully accomplished. The dependence of the polarization handedness as a function of the phase between the two adjacent undulators was measured and found to be in good agreement with theory, which indicates that scaling crossedplanar undulators to seeded $\mathrm{x}$-ray FELs may be possible.

The design considerations, commissioning, and the experimental details of the polarization switching at
SDUV-FEL are discussed in Refs. [12-13]. In the experiment, the linear accelerator provides an electron beam of $145 \mathrm{MeV}$ with the bunch charge of $150 \mathrm{pC}$, normalized emittance less than $4 \mathrm{~mm}$-mrad, projected energy spread of $0.2 \%$, and pulse duration of approximately 8 ps FWHM without compression. The layout of the polarization switching experiment is schematically shown in Fig. 1. The main components consist of an electromagnetic planar modulator (EMU), a dispersive chicane (DS) and a crossed-planar radiator system (PMU-H, PS, PMU-V), several YAG/ optical transition radiation (OTR) screens for electron and laser beam position and size measurements, correctors for beam trajectory control, and quadrupoles for beam matching and focusing. The EMU (10 periods, period length of $65 \mathrm{~mm}$, and variable undulator magnetic field) and PMU-H (10 periods, period length of $50 \mathrm{~mm}$, and alterable undulator gap) previously served as the twin modulator in an echo-enabled harmonic generation FEL lasing experiment at SDUV-FEL [35]. In order to conduct the polarization switching experiment, a small electromagnetic phase shifter, PS with maximum $R_{56}$ of $5 \mu \mathrm{m}$, a vertical planar undulator, PMU-V (10 periods, period length of $50 \mathrm{~mm}$, and alterable undulator gap), and an optical bench for FEL radiation measurements were added at the downstream of PMU-H.

The seed laser is from the $1047 \mathrm{~nm}$ drive laser (8.7 ps FWHM pulse length), with tunable pulse energy between 0 and $100 \mu \mathrm{J}$. The electron and laser beam are overlapped spatially and temporally in the modulator, and the dispersive strength optimized $\left(R_{56}=3 \mathrm{~mm}\right.$ in the experiment). The microbunched electron beam is then sent through the crossed-planar radiators, which are tuned to be resonant at the second harmonic of the seed laser, to produce coherent FEL radiation at the central wavelength of $523 \mathrm{~nm}$. Using the modified optical replica method [36-37], critical parameters for the density modulation, such as the sliced beam energy spread and the energy modulation amplitude induced by the seed laser, were precisely quantified. In this experiment, the sliced beam energy spread and the energy modulation amplitude were measured to be 1.2 and $12 \mathrm{keV}$, respectively, which corresponds to a strong bunching factor over $40 \%$ for $523 \mathrm{~nm}$ radiation at the entrance of the radiators. Since the phase-shifter dispersion is small and both the crossed radiators are only 10 periods, the electron beam can be treated as a rigid microbunched beam. Therefore, PMU-H 
and PMU-V should deliver identical radiation pulses with nearly equivalent power and phase but mutually perpendicular linear polarization. Then the phase shifter can be used to fine-tune the path difference between the horizontal and vertical polarized pulses, and hence realize polarization switching of the combined radiation.

The polarization of the FEL light was characterized using a division-of-amplitude photopolarimeter (DOAP) that was constructed in house. It consists of one optical lens, three beam splitters, four polarizers, one quarter-wave plate, and four photodetectors. The incident light is divided into four separate beams, and the Stokes parameters $\left(S_{0}, S_{1}, S_{2}, S_{3}\right)$ measured simultaneously, thus allowing one to fully determine the polarization of the light emitted from the crossed-planar radiators in a single shot. The DOAP was intensively calibrated using the frequencydoubled $(523 \mathrm{~nm}) \mathrm{Nd}$ :YLF laser. Here we recall that $S_{1}$ represents the fraction of intensity linearly polarized; $S_{2}$ is similar to $S_{1}$ but in a reference system rotated by $45^{\circ}$; and $S_{3}$ is the fraction of intensity showing circular polarization. In particular, one finds $S_{1} / S_{0}= \pm 1$ when the polarization is fully linear horizontal (vertical), while $S_{3} / S_{0}= \pm 1$ for right-handed (left-handed) circular polarization. In order to test the setup, the seed laser was turned off and the PMU-V gap was changed from the maximum to $35 \mathrm{~mm}(523 \mathrm{~nm}$ resonance) while the PMU-H was kept on resonance at $523 \mathrm{~nm}$, then a set of measurements of the combined spontaneous emission was performed. As seen from the normalized Stokes's parameters $S_{1} / S_{0}, S_{2} / S_{0}$, and $S_{3} / S_{0}$ plotted in Fig. 2, when the undulator gap of PMU-V was fully opened, the light was mainly emitted from the PMU-H and completely horizontally polarized. As the vertical field component from the PMU-V was enhanced, the linear polarization degree of the combined spontaneous radiation decreases monotonously. For the case that both

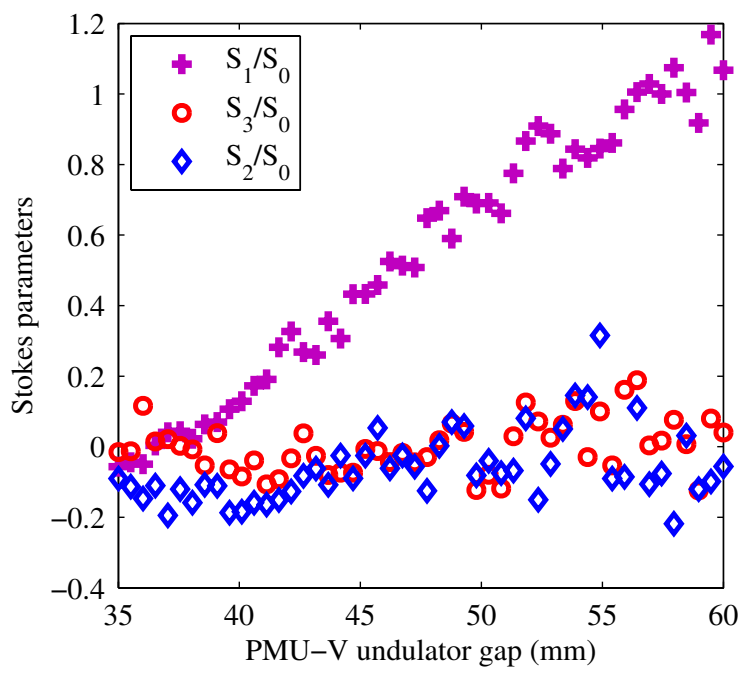

FIG. 2 (color online). The polarization performance of the combined spontaneous emission of crossed-planar undulators, characterized by the DOAP system. crossed-planar radiators are set to be resonant at $523 \mathrm{~nm}$, the combined spontaneous emission is completely unpolarized, even though they were perfectly synchronized spatially and temporally. This expected behavior depends on the fact that the coupling between the random phases of the vertical and horizontal spontaneous emission results in an arbitrary orientation for the combined light's electric field. This test actually presents the crossed-planar undulators experimental result on storage ring light sources without a monochromator, which demonstrates that the longitudinal coherence of the crossed lights is important and essential for the crossed-planar undulators technique.

After optimizing all of the undulator resonance conditions, and the beam orbit in the polarization switching beam line, the seed laser was turned on and the coherent $523 \mathrm{~nm}$ radiation pulses generated from the crossed-planar undulators were reflected out of the vacuum chamber by a OTR screen and guided into an Ocean QE65000 spectrometer that is capable of detecting the signal from 350 to $1100 \mathrm{~nm}$. By either kicking away the electron beam after PMU-H or opening up the gap of PMU-V, the coherent radiation pulses generated in the two undulators could be sent into the spectrometer separately. Since the electron beam was accelerated on the crest in the linear accelerator, the linear beam energy chirp was minimized; thus, both the vertical and horizontal polarized FELs were centered on $523 \mathrm{~nm}$ wavelength, as indicated in Fig. 3, which shows that the two crossed lights with the same frequency are ready for effective combination and polarization switching. One should note that the spectra bandwidths are broader than the Fouriertransform-limited case, due to the limited spectrometer resolution of $2.4 \mathrm{~nm}$, and the residual energy curvature from the varying rf phase along the 8 ps electron bunch.

It was then attempted to switch and characterize the polarization of coherent $523 \mathrm{~nm}$ radiation by adjusting the phase shifter. With a relatively stable beam and optimized experimental conditions, the measured Stokes parameters were as given in Fig. 4. The circular polarization of the combined FEL was increased from $20 \%$ to $80 \%$, while the linear polarization $\left(S_{2}\right)$ was reduced from $95 \%$ to $20 \%$. As a consequence, the combined FEL remained almost fully polarized during tuning. When we further increased the phase-shifter strength, the switching between linear polarization and circular polarization was reproduced reasonably.

Generally speaking, one of the most important points for achieving perfect polarization switching is that the radiation pulses from the two crossed undulators should be as close to identical as possible. In the experiment, we can slightly tune the gaps of crossed undulators and the quadrupoles to obtain almost equivalent FEL powers, while the radiation phase is determined by the seed laser. The other important point is that the radiation pulses should be both temporally and spatially overlapped. Because of the FEL slippage effect in the crossed-planar undulators system, the temporal overlap of the $523 \mathrm{~nm}$ radiation pulses 

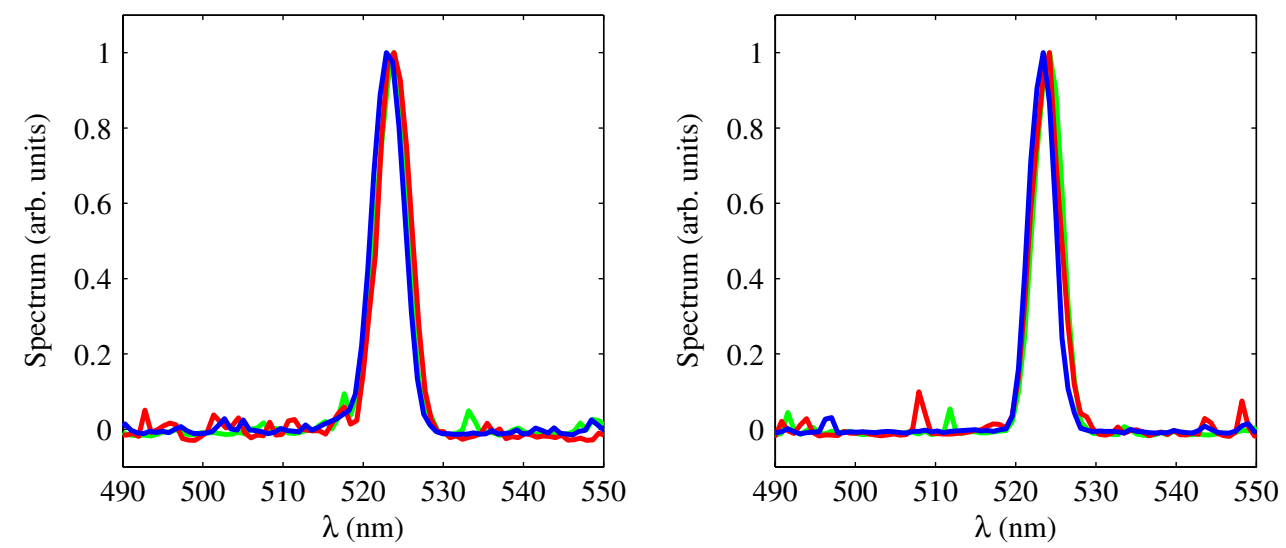

FIG. 3 (color online). Three typical shots of $523 \mathrm{~nm}$ radiation spectra from the crossed-planar undulators, where the signal was normalized to the peak value. (a) Radiation with horizontally linear polarization generated in PMU-H. (b) Radiation with vertically linear polarization generated in PMU-V.

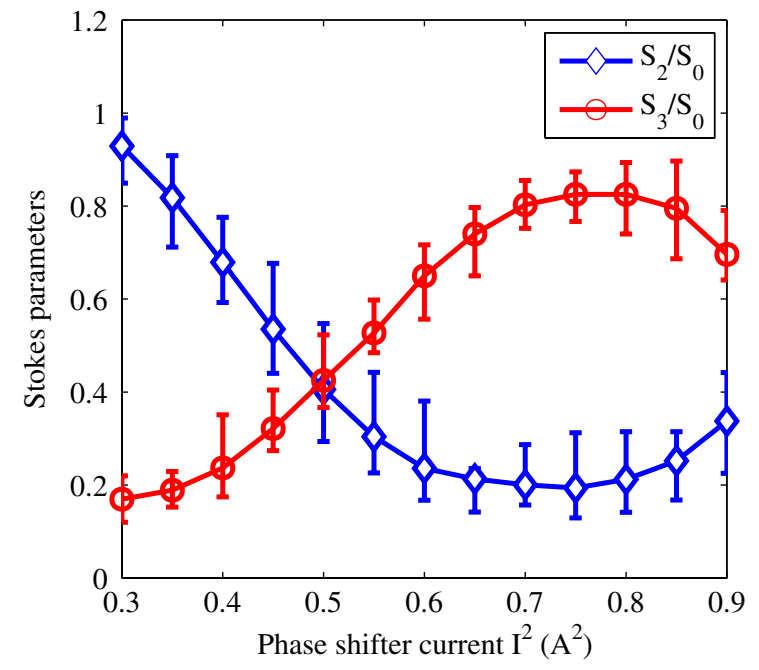

FIG. 4 (color online). The normalized Stokes parameters of coherent $523 \mathrm{~nm}$ radiation measured at SDUV-FEL, where the phase-shifter current square is tuned from 0.3 to 0.9 , corresponding to a phase delay of approximately $0.6 \pi$.

from PMU-H and PMU-V is naturally imperfect. It has been identified using start-to-end simulations that the imperfect temporal overlap, together with the inevitable residual beam energy chirp at SDUV-FEL, will degrade the polarization performance of the combined FEL radiation pulses by a factor of $10 \%$ [12]. Figure 5 shows two typical transverse profiles of the radiation pulses captured on a charge-coupled device (CCD) in our experiment. In order to clearly illustrate the effects of the spatial overlap, simulations using the two profiles were carried out. It is found that the polarization degree approaches $90 \%$ when the two radiation pulses are optimally overlapped, while it decreases to $50 \%$ when the two radiation pulses are separated by $200 \mu \mathrm{m}$. The spatial overlap is determined by the beam orbit and envelope within the undulators, and

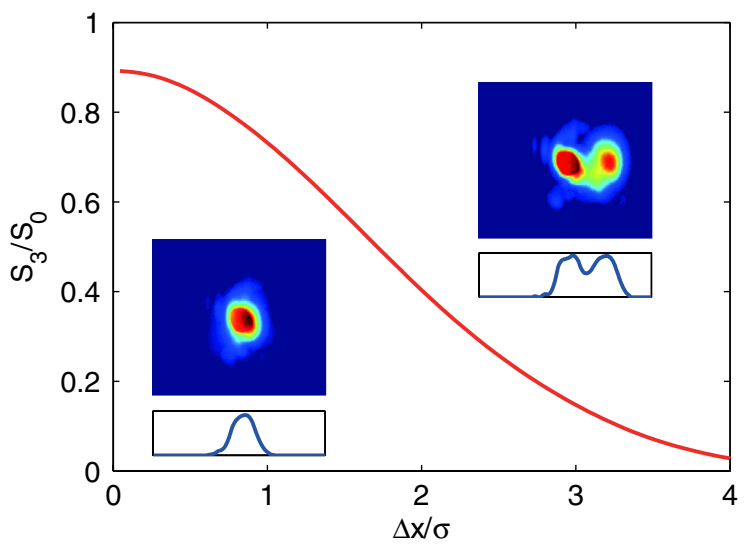

FIG. 5 (color online). Simulated polarization performance of the combined $523 \mathrm{~nm}$ radiation vs the transverse position discrepancy of the two crossed radiation pulses where the experimentally observed transverse profiles are used.

the propagation in downstream free space. In the experiment, a shot-to-shot jitter of the photon beam position was clearly observed on the CCD camera. The large polarization fluctuations in Fig. 4 were mainly contributed by the beam instability at SDUV-FEL due to the lack of a feedback system.

In summary, using a seeded FEL configuration at SDUVFEL, we presented the first experimental demonstration of polarization switching by crossed-planar undulators. The good agreement between the theory and our experimental results confirms the physics behind this technique. It can be easily extended to the existing [2,5] and future seeded FEL facilities [38-40] aimed from extreme ultraviolet to a soft $\mathrm{x}$-ray spectral region, in which fully coherent radiation pulses with fast polarization switching are expected to open up new scientific opportunities in various research fields. It is worth stressing that, in a short-wavelength FEL, the polarization degradation due to the electron beam 
instability and radiation diffraction would be significantly improved in comparison with the current experiment accomplished at visible wavelength. In this proof-of-principle experiment, the linear accelerator repetition rate of $2 \mathrm{~Hz}$ limits the maximum polarization switching rate to $1 \mathrm{~Hz}$, which, however, is already an order of magnitude faster than those techniques that require mechanical adjustments of undulator magnets. Moreover, the greatest advantage of the crossed-planar undulators for fast polarization switching will definitely benefit the future high repetition rate FEL facilities, e.g., the Next Generation Light Source proposed in the United States [40].

The authors would like to thank Y. Li, B. Faatz, Y. Ding, Z. Huang, A. Chao, K. Kim, H. Geng, Q. Jia, and Z. Dai for helpful suggestions, J. Yan and D. Dunning for useful discussions and improving the writing, and the SDUV-FEL team for experiment setup establishment and commissioning assistance. This work was partially supported by the Major State Basic Research Development Program of China (No. 2011CB808300) and the National Natural Science Foundation of China (No. 11175240, No. 11205234, and No. 11322550).

[1] B. W. J. McNeil and N. R. Thompson, Nat. Photonics 4, 814 (2010).

[2] W. Ackermann et al., Nat. Photonics 1, 336 (2007).

[3] P. Emma et al., Nat. Photonics 4, 641 (2010).

[4] T. Ishikawa et al., Nat. Photonics 6, 540 (2012).

[5] E. Allaria et al., Nat. Photonics 6, 699 (2012).

[6] C. Spezzani, E. Allaria, M. Coreno, B. Diviacco, E. Ferrari, G. Geloni, E. Karantzoulis, B. Mahieu, M. Vento, and G. De Ninno, Phys. Rev. Lett. 107, 084801 (2011).

[7] K. J. Kim, Nucl. Instrum. Methods Phys. Res., Sect. A 219 , 425 (1984).

[8] K. J. Kim, Nucl. Instrum. Methods Phys. Res., Sect. A 445, 329 (2000).

[9] Y. Ding and Z. Huang, Phys. Rev. ST Accel. Beams 11, 030702 (2008).

[10] Y. Li, B. Faatz, and J. Pflueger, Nucl. Instrum. Methods Phys. Res., Sect. A 613, 163 (2010).

[11] H. Geng, Y. Ding, and Z. Huang, Nucl. Instrum. Methods Phys. Res., Sect. A 622, 276 (2010).

[12] T. Zhang et al., Nucl. Instrum. Methods Phys. Res., Sect. A 680, 112 (2012).

[13] H. Deng et al., in Proceeding of 34th International Free Electron Laser Conference (FEL12), Nara, Japan, 2012, pp. 245-248 (unpublished).

[14] J. Bahrdt, A. Gaupp, W. Gudat, M. Mast, K. Molter, W. B. Peatman, M. Scheer, Th. Schroeter, and Ch. Wang, Rev. Sci. Instrum. 63, 339 (1992).
[15] R. Reininger, C. SánchezHanke, and S. L. Hulbert, AIP Conf. Proc. 1234, 383 (2010).

[16] K. Tsuchiya, T. Shioya, T. Aoto, K. Harada, T. Obina, M. Sakamaki, and K. Amemiya, J. Phys. Conf. Ser. 425, 132017 (2013).

[17] Y. K. Wu, N. A. Vinokurov, and S. Mikhailov, Phys. Rev. Lett. 96, 224801 (2006).

[18] L. H. Yu, Phys. Rev. A 44, 5178 (1991).

[19] J. Wu and L. H. Yu, Nucl. Instrum. Methods Phys. Res., Sect. A 475, 104 (2001).

[20] G. Stupakov, Phys. Rev. Lett. 102, 074801 (2009).

[21] H. Deng and C. Feng, Phys. Rev. Lett. 111, 084801 (2013).

[22] J. Amann et al., Nat. Photonics 6, 693 (2012).

[23] D. Xiang, Y. Ding, Z. Huang, and H. Deng, Phys. Rev. ST Accel. Beams 16, 010703 (2013).

[24] B. W. J. McNeil, N. R. Thompson, and D. J. Dunning, Phys. Rev. Lett. 110, 134802 (2013).

[25] J. Dai, H. Deng, and Z. Dai, Phys. Rev. Lett. 108, 034802 (2012).

[26] Z. Huang, Y. Ding, and C. B. Schroeder, Phys. Rev. Lett. 109, 204801 (2012).

[27] C. Chang, C. Tang, and J. Wu, Phys. Rev. Lett. 110, 064802 (2013).

[28] T. Zhang et al., arXiv:1304.7420.

[29] A. A. Lutman, R. Coffee, Y. Ding, Z. Huang, J. Krzywinski, T. Maxwell, M. Messerschmidt, and H.-D. Nuhn, Phys. Rev. Lett. 110, 134801 (2013).

[30] E. Allaria et al., Nat. Commun. 4, 2476 (2013).

[31] M. Suzuki, N. Kawamura, M. Mizumaki, A. Urata, H. Maruyama, S. Goto, and T. Ishikawa , J. Synchrotron Radiat. 6, 190 (1999).

[32] C. Sanchez-Hanke, D. Lott, J. Stohr, and C. C. Kao, Rev. Sci. Instrum. 73, 1639 (2002).

[33] C. Sanchez-Hanke, C. C. Kao, and S. L. Hulbert, Nucl. Instrum. Methods Phys. Res., Sect. A 608, 351 (2009).

[34] Z. T. Zhao, Z. M. Dai, X. F. Zhao, D. K. Liu, Q. G. Zhou, D. H. He, Q. K. Jia, S. Y. Chen, and J. P. Dai, et al., Nucl. Instrum. Methods Phys. Res., Sect. A 528, 591 (2004).

[35] Z. T. Zhao et al., Nat. Photonics 6, 360 (2012).

[36] C. Feng, T. Zhang, J. Chen, H. Deng, M. Zhang, X. Wang, B. Liu, T. Lan, D. Wang, and Z. Zhao, Phys. Rev. ST Accel. Beams 14, 090701 (2011).

[37] H. Deng, C. Feng, B. Liu, D. Wang, X.-T. Wang, and M. Zhang, Chin. Phys. Lett. 28, 124101 (2011).

[38] J. Yan, M. Zhang, and H. Deng, Nucl. Instrum. Methods Phys. Res., Sect. A 615, 249 (2010).

[39] T. Zhang, H.-X. Deng, W.-Q. Zhang, G.-R. Wu, D.-X. Dai, D. Wang, X.-M. Yang, and Z.-T. Zhao, Chin. Phys. C 37, 118101 (2013).

[40] Report of the BESAC Subcommittee on Future X-ray Light Sources, 2013, http://science.energy.gov. 